\title{
Involvement of endothelin B receptor in peripheral nerve regeneration using sciatic nerve transection-regeneration model
}

\author{
Nguyen Huu Tu, Tayo Katano, Shinji Matsumura, and Seiji Ito \\ Department of Medical Chemistry, Kansai Medical University
}

\begin{abstract}
Whereas the central nervous system usually cannot regenerate, axons within the peripheral nervous system regenerate spontaneously and rapidly after injury. To study molecular mechanisms of peripheral nerve regeneration, we established the sciatic nerve transection-regeneration model by using a combination of tabulation and osmotic mini-pump to deliver drugs at a constant rate for at least 4 weeks and have recently demonstrated that endothelin (ET)-1 was involved in the nerve regeneration. In the present study, to clarify which receptors of ET-1 mediated its action, we examined the effect of ETAR and ETBR antagonists on the functional recovery. Mice started to resume mechanical responses of their hind paws 5 weeks after the sciatic nerve transection and returned to the level before operation by 7 weeks. Time course of the functional recovery is well correlated with that of nerve regeneration assessed by elongation of axons expressing yellow fluorescent protein and application of fluoro-ruby, a fluorescent retrograde tracer, to the distal stump of the transected nerve. The ETвR antagonist, but not ETAR antagonist, delayed the functional recovery in the model mice. Taken together with ETвR expression in Schwann cells, these results demonstrate that ET-1 is involved in peripheral nerve regeneration via ETвR.
\end{abstract}

Keywords

Endothelin; Endothelin receptor type B (ЕTвR); Functional recovery; Nerve regeneration; Peripheral nervous system; thy1-YFP mice

Received: 4 December 2014

Accepted: 22 January 2015 


\section{Introduction}

Although causalgia (complex regional pain syndrome type II) was considered to be incurable since its first description in 1864, it was reported that two patients with causalgia were treated by nerve reconstruction surgery using in situ tissue engineering with collagen tube ${ }^{1)}$. Prior to nerve reconstruction, functional regeneration requires axon regrowth and remyelination of the regenerated axons by Schwann cells under a permissive environment and activation of the intrinsic growth capacity of neurons. To understand the molecular basis of nerve regeneration, we established the sciatic nerve transectionregeneration model ${ }^{7}$. We have recently demonstrated that the functional recovery was delayed in mice lacking $\mathrm{Nax}$, a sodium concentrationsensitive sodium channel and that $\mathrm{Nax}$ is functionally coupled to endothelin-1 (ET-1) for lactate release and is involved in peripheral nerve regeneration ${ }^{8)}$. ET-1 is a peptide with 21 amino acids and exerts diverse biological actions via two ET receptors type A (ETAR) and type $B$ $(\mathrm{ET} \mathrm{BR})^{3)}$. By in vitro experiments using cultured Schwann cells, we demonstrated that lactate release was enhanced by ET-1 and blocked by an ETвR antagonist. However, whether ET-1 is involved in peripheral nerve regeneration in vivo remains unclarified. To address this question, we studied the effect of antagonists of ETAR and ETвR on functional recovery using our model.

\section{Materials and Methods}

\section{Animal and surgery}

Animal experiments were approved by the Animal Experimentation Committee, Kansai Medical University, and carried out in accordance with the National Institutes of Health guide for the care and use of laboratory animals. Thy1-yellow fluorescent protein (thy1-YFP) transgenic [B6.Cg-Tg (thy1-YFP) 16Jrs/J] mice were purchased from Jackson Laboratory, and housed and bred in Laboratory Animal Center of Kansai Medical University under conditions of a 12-h light : 12 -h dark cycle, a constant temperature of $22 \pm 2^{\circ} \mathrm{C}$ and $60 \pm 10 \%$ humidity. Male 8-week-old thy1-YFP transgenic and littermate wild-type mice weighing from 22 to $27 \mathrm{~g}$ were subjected to the sciatic nerve transection-regeneration model as recently reported ${ }^{7,8)}$. In brief, the right sciatic nerve was transected at the mid-thigh level and the nerve stumps were sutured to a silicone tube, leaving a $5-\mathrm{mm}$ gap and filling with physiological saline. A catheter tube (7701; Alzet, Cupertino, CA, USA) was attached to an osmotic pump (1004; Alzet) pre-filled with $1 \mu \mathrm{M}$ ETAR antagonist BQ-123, ETвR antagonist BQ-788 (EMD Millipore, San Diego, CA, USA) or phosphate-buffered saline (PBS). The pump was subcutaneously implanted on the back and the osmotic pump-catheter system released medications into the silicone chamber constantly for 4 weeks.

\section{Labeling DRG neurons with fluoro-ruby (F-ruby)}

To examine sciatic nerve regeneration, $\mathrm{F}-$ ruby (dextran, tetramethylrhodamine, 10,000 MW, Life Technologies, Tokyo, Japan) was used. Before and 2, 4, 8 weeks after axotomy, the wound was re-opened, the distal site of the 
transected sciatic nerve was exposed, and then $10 \%$ F-ruby $(5 \mu \mathrm{l})$ mixed in gel foam ${ }^{\circledR}$ (Pfizer, Tokyo, Japan) was put on the re-transected site peripheral to the distal stump of the sciatic nerve. After $30 \mathrm{~min}$, the gel was removed. Three days after the treatment, L4-6 DRGs were isolated. F-ruby-labeled DRG neurons were counted in a whole-mount DRG obtained by preparing 13 - 22 image stacks ( $25-\mu \mathrm{m} \mathrm{z}$ steps) by a Zeiss LSM 510 laser scanning confocal microscope.

\section{Behavioral testing}

To check the functional recovery, we measured withdrawal responses to mechanical stimulus on the hind paw, using calibrated von Frey filaments starting from $0.4 \mathrm{~g}$. Behavioral tests were conducted before and every week after the operation for 8 weeks. The mechanical force of $4 \mathrm{~g}$ was used as a cut-off value of withdrawal thresholds.

\section{Imunohistochemistry}

Immunostaining was carried out according to the procedure described previously ${ }^{8)}$. The nerve segments were frozen in OCT compound (Leica Microsystems, Tokyo, Japan) and cut into $10-\mu \mathrm{m}$ transverse sections on a cryostat. The sections were incubated for $1 \mathrm{~h}$ at room temperature in a blocking solution (10\% normal goat serum and $0.1 \%$ Triton X-100 in PBS), and then incubated with rabbit anti-ETвR (1:100 Novus Biologicals, Littleton, CO, USA) and mouse anti NF-200 monoclonal antibody (1: 500, Sigma) overnight at $4^{\circ} \mathrm{C}$. After being washed with PBS, the sections were then treated by fluorescent-conjugated secondary antibodies: Alexa 594-goat anti rabbit IgG (1:300, Invitrogen, Carlsbad, CA, USA) and alexa 488-goat anti mouse IgG (1:300, Invitrogen) for $2 \mathrm{~h}$ at room temperature. The fluorescent images were obtained with a Zeiss
LSM 510 META laser scanning confocal microscope.

\section{Results}

\section{Characterization of sciatic nerve regeneration}

Figure 1-A shows the sciatic nerve transection-regeneration model. To ease the evaluation of nerve regeneration, we utilized thy1-YFP mice selectively expressing YFP in neurons. YFP was transported from the soma and accumulated in the proximal stump 1 week after the operation (Fig.1-B), and it was observed in the regenerated nerves 4 weeks (Fig.1-C). The regenerated nerve elongated and reached the foot 8 weeks after the operation (Fig.1-D, E). To confirm the sciatic nerve regeneration, we re-opened the wound and applied F-ruby, a retrograde nerve tracer, on the distal site of the regenerated sciatic nerve before, 2,4 , and 8 weeks after the transection (Fig.1-F). The number of F-ruby-labeled DRG neurons was increased time-dependently for 8 weeks after axotomy, being 525, 1406, 1656 cells / mouse at 2, 4, and 8 weeks, respectively (Fig.1-G, H). Assuming that the number of DRG neurons was constant, $26.5,70.7$ and $83.3 \%$ of regenerated nerves reached the distal side 2,4 , and 8 weeks after axotomy.

\section{Involvement of $E T_{B} R$ in functional recovery}

To examine which ET receptors were involved in the peripheral nerve regeneration, we studied the effect of ETAR and ETBR antagonists on the functional recovery by the withdrawal responses to mechanical stimuli. The hind paw with- 


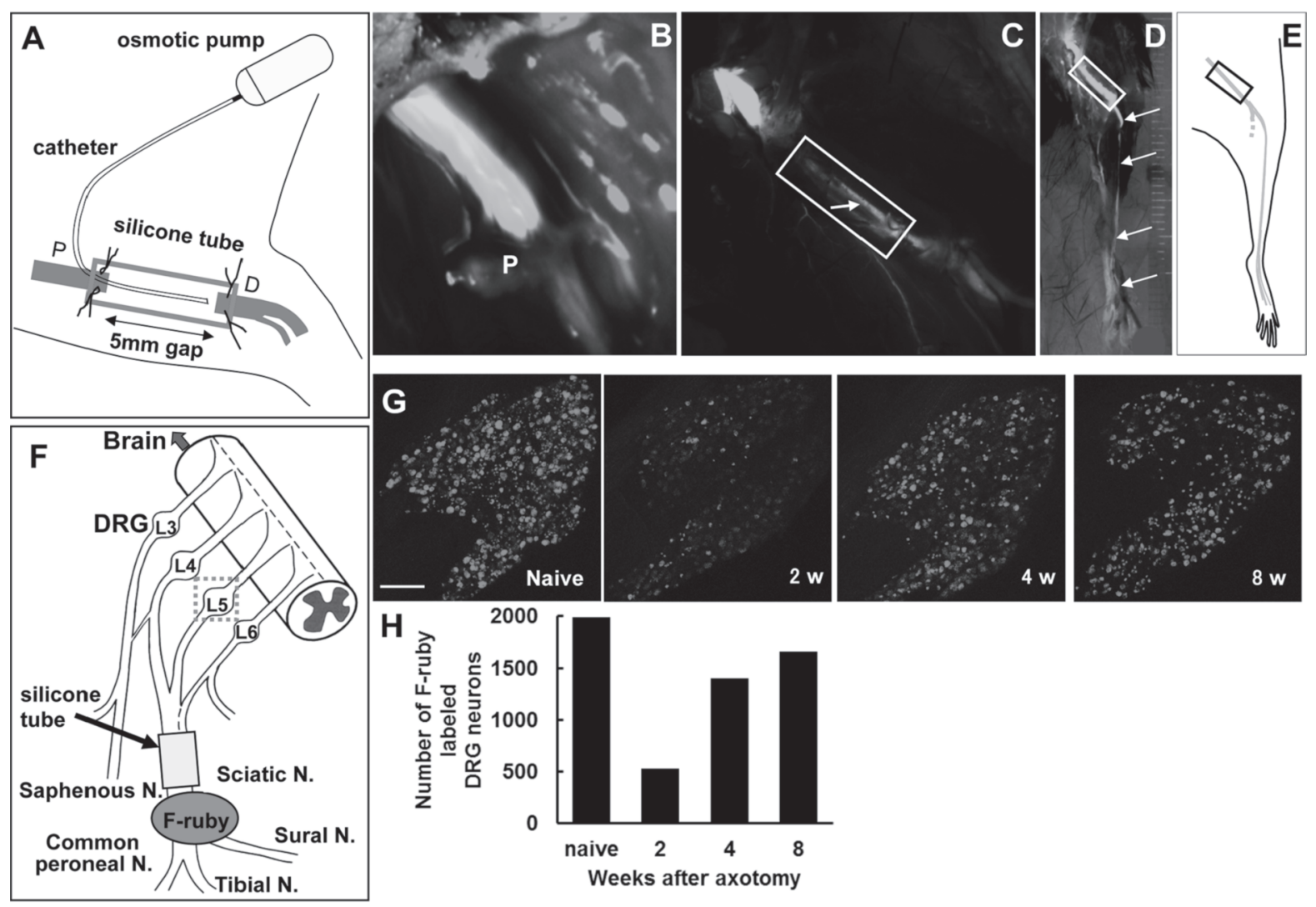

Fig.1 Re-innervation of sciatic nerve after transection.

(A) Diagram of the sciatic nerve transection-regeneration model. Proximal (P) and distal (D) transected stumps were sutured to the inner wall of a silicone tube. A drug was continuously delivered into the silicone tube from an osmotic pump for 4 weeks.

(B-D) Fluorescent microscopic imaging of YFP-labeled sciatic nerve 1 week (B), 4 weeks (C), and 8 weeks (D) after the operation. (E) Diagram of fluorescent image of the lower limb. Arrows indicate regenerated nerves. (F-H) Retrograde transport of F-ruby, a fluorescent tracer to DRG. (F) The diagram of labeling L5 DRG neurons by F-ruby. Five $\mu \mathrm{L}$ of $10 \% \mathrm{~F}$-ruby $(10,000 \mathrm{MW})$ were put close to the distal (D) stump of the transected sciatic nerve before and 2, 4, and 8 weeks after the operation. Three days after F-ruby loading, L4-L6 DRGs were observed by confocal microscopy. (G) Fluorescent images of L5 DRG neurons and scale bar $=250 \mu \mathrm{m}$. (H) Quantification of F-ruby-labeled DRG neurons.

drawal thresholds to mechanical stimuli before operation were 0.4 to $0.6 \mathrm{~g}$. When the responses were measured every week after the operation, none of the mice responded to the cut-off stimulus of $4.0 \mathrm{~g}$ for the first 4 weeks. All mice in the control group responded to mechanical stimulation at the $5^{\text {th }}$ week and returned to the pre-operation level at the $7^{\text {th }}$ week (Fig.2-A). The ETвR antagonist, but not the ETAR antagonist, delayed the functional recovery by 2 weeks (Fig.2-A). These results suggest that ET-1 is involved in peripheral nerve recovery though the ETвR receptor.

The transverse section of the sciatic nerve was immunostained with NF-200, a marker of myelinated axons. ETвR was expressed around axons with a typical round form of myelinating Schawnn cells (Fig.2-B). 

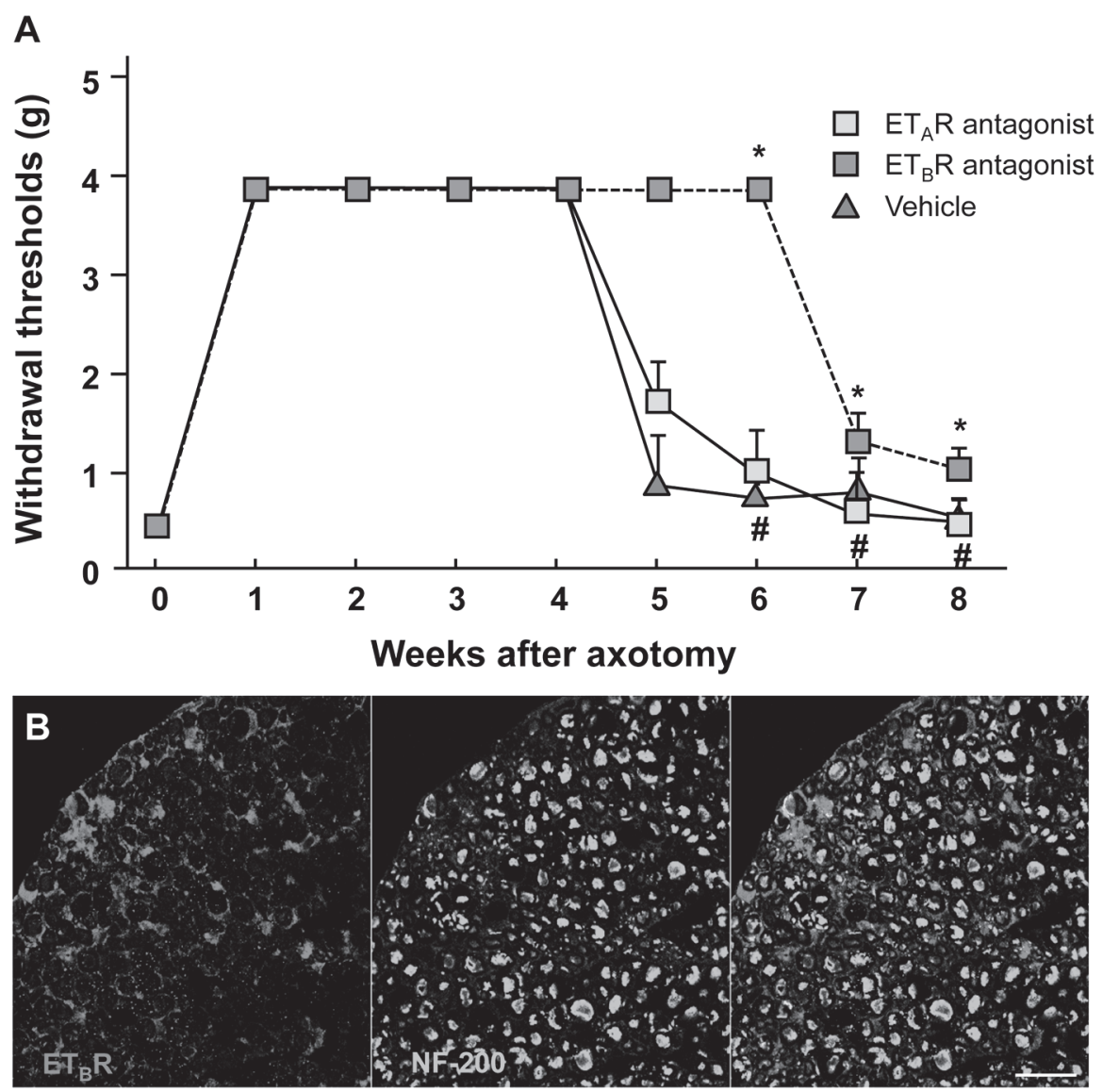

Fig.2 Involvement of ETвR in peripheral nerve regeneration.

(A) Effect of ET receptor antagonists on the functional recovery from sciatic nerve transection. PBS (vehicle), $1 \mu \mathrm{M}$ ETAR antagonist or $1 \mu \mathrm{M}$ ETBR antagonist was continuously delivered into the silicone tube from an osmotic pump immediately after the operation. The recovery was assessed every week before and after the operation by paw withdrawal thresholds to von Frey filaments. The mechanical force of $4 \mathrm{~g}$ was used as a cut-off value of withdrawal thresholds. Data show the mean $\pm \mathrm{SD}(n=7-8) .{ }^{*} p<0.05$ vs vehicle group, by Mann-Whitney's U test.

(B) ETвR expression in the sciatic nerve. Transverse sections of the naïve sciatic nerve was immunostained with anti-ETвR (red) and anti-NF-200 (green) antibodies. Double labeling was created by merging fluorescent images of ETBR and NF-200. Scale bar $=20 \mu \mathrm{m}$.

\section{Discussion}

The sciatic nerve transection-regeneration model using a combination of tubulation and osmotic mini-pump enabled us to deliver drugs into the silicone tube through a catheter at a constant rate for at least 4 weeks. To study whether 4 weeks for drug administration are a period long enough to evaluate its effect, we first characterized nerve regeneration by thy1YFP mice and F-ruby, a retrograde tracer. Consistent with YFP-labeled nerve elongation (Fig.1-B, C, D), retrograde F-ruby-labeling showed about $70 \%$ of regenerated axons reached the distal side of the transected nerve at the $4^{\text {th }}$ week (Fig.1-G, H). These results demonstrate that a period of 4 weeks is enough 
to evaluate the effect of a drug on the initial nerve regeneration after axotomy.

The histochemical observations agree with the functional recovery after sciatic nerve transection; it resumed at the 5th week and return to the level before operation at the 7 th week (Fig.2-A). This functional recovery was delayed by two weeks with the ETвR antagonist, but not with the ETAR antagonist (Fig.2-B). These in vivo results that ET-1 promoted the nerve regeneration via ETвR support the in vitro experiments using cultured Schwann cells ${ }^{8)}$. Whether the delay in the sensory function by two weeks is ascribed to the drug itself or the capacity of osmotic mini-pump remains unclarified.

Schwann cells are categorized into two groups based on the myelinating ability, i.e. myelinating and non-myelinating Schwann cells ${ }^{2)}$. Although we hypothesized that ET-1 is coupled to Nax for lactate release in nonmyelinating Schwann cells ${ }^{8)}$, ETвR was mainly expressed in myelinated Schawann cells surrounding axons in the sciatic nerve (Fig.2-B). ETBR was previously shown to be expressed in non-myelinated Schwann cells in sciatic nerve and satellite cells in the DRG, whereas ETAR was expressed in small-sized sensory neurons ${ }^{5)}$. Furthermore, ET-1 was locally up-regulated at the injury site after nerve injury ${ }^{4,6}$. We need further investigation concerning the expression of ETвR after nerve transection.

In conclusion, here we demonstrate a novel role of the ET system in nerve regeneration, in addition to nociception and pain in the peripheral nervous system in the literature.

\section{Acknowledgements}

This study was partially supported by grants (22390063, 2010 and 24220010, 2012) from the program Grants-in-Aid for Scientific Research
(B) and (C) of the Ministry of Education, Culture, Sports, Science, and Technology of Japan.

\section{References}

1) Inada, Y., Morimoto, S., Moroi, K., Endo, K., Nakamura, T., Surgical relief of causalgia with an artificial nerve guide tube: Successful surgical treatment of causalgia (Complex Regional Pain Syndrome Type II) by in situ tissue engineering with a polyglycolic acid-collagen tube, Pain, 117 (2005) 251-258.

2) Jessen, K.R., Mirsky, R., The origin and development of glial cells in peripheral nerves, Nat. Rev. Neurosci., 6 (2005) 671-682.

3) Kedzierski, R.M., Yanagisawa, M., Endothelin system: the double-edged sword in health and disease, Annu. Rev. Pharmacol., 41 (2001) 851876.

4) Klass, M., Hord, A., Wilcox, M., Denson, D., Csete, M., A role for endothelin in neuropathic pain after chronic constriction injury of the sciatic nerve, Anesth. Analg., 101 (2005) 1757-1762.

5) Pomonis, J.D., Rogers, S.D., Peters, C.M., Ghilardi, J.R., Mantyh, P.W., Expression and localization of endothelin receptors: implications for the involvement of peripheral glia in nociception, J. Neurosci., 21 (2001) 999-1006.

6) Rae, G.A., Henriques, M.G., Endothelins in inflammation. In: Said, S. (Ed.), Pro-inflammatory and anti-inflammatory peptides, Marcel \& Dekker, New York, 1998, pp163202.

7) Unezaki, S., Yoshii, S., Mabuchi, T., Saito, A., Ito, S., Effects of neurotrophic factors on nerve regeneration monitored by in vivo imaging in thyl-YFP transgenic mice, J. Neurosci. Methods, 178 (2009) 308-315.

8) Unezaki, S., Katano, T., Hiyama, Y., Nguyen, H.T., Yoshii, Y., Noda, M., Ito, S., Involvement of Nax sodium channel in peripheral nerve regeneration via lactate signaling, Eur. J. Neurosci., 39 (2014) 720-729.

Address for correspondence: Seiji Ito

Department of Medical Chemistry,

Kansai Medical University

2-5-1 Shinmachi, Hirakata, Osaka 573-1010, Japan

Tel: +81-72-804-2340

E-mail: ito@hirakata.kmu.ac.jp 